DOI: https://doi.org/10.46296/yc.v4i7edespdic.0076

\title{
PLAN DE MEJORA EN CALIDAD DEL SERVICIO ALIMENTOS Y BEBIDAS DEL HOTEL MELIÁ CAYO COCO
}

\section{PLAN TO IMPROVE THE QUALITY OF THE FOOD AND BEVERAGE SERVICE AT THE HOTEL MELIÁ CAYO COCO}

\author{
Hernández-Blanco Jovel ${ }^{1 *}$; Urgellés-Reyes Mario²; Wilson-González Fabian Enrique; \\ Loredo-Carballo Néstor ${ }^{4}$ \\ ${ }^{1}$ Especialista de gestión de los servicios de alimentos y bebidas, Hotel Melia Cayo \\ Coco. Ciego de Ávila, Cuba. Orcid: https://orcid.org/0000-0002-0921-0798 \\ 2 Universidad de Camagüey Ignacio Agramonte Loynaz. Camagüey, Cuba. \\ ${ }^{3}$ Universidad de Camagüey Ignacio Agramonte Loynaz. Camagüey, Cuba. Orcid: \\ https://orcid.org/0000-0002-7984-944X \\ ${ }^{4}$ Profesor Universidad de Camagüey Ignacio Agramonte Loynaz. Camagüey, Cuba.
}

*Correo: hbjovel@yahoo.com

\section{Resumen}

Se propone un plan de mejora de la calidad del servicio de Alimentos y Bebidas en el Hotel Meliá Cayo Coco, mediante la aplicación del método ServQual que permitió tomar decisiones a partir de la percepción y expectativa del cliente sobre el servicio. Para tal efecto, se construyó un cuestionario que recogió 19 indicadores que representan las cinco dimensiones señaladas por Vera y Causado, medidos en una escala Likert de 5 puntos, el cual fue aplicado a 374 clientes, tomando en cuenta el valor mensual más alto correspondiente a la cantidad de clientes que accedieron al servicio, durante el trimestre precedente al mes fiscal objeto de evaluación. Los niveles de expectativas más elevados se encontraron en las dimensiones: fiabilidad, responsabilidad y bienes tangibles, mientras que aquellas percibidas como de mejor calidad fueron seguridad y empatía. En general, los niveles de calidad del servicio se mostraron desfavorables o negativos debido principalmente a que no fueron superadas las expectativas de los clientes por sus percepciones, información que facilitó realizar acciones para alcanzar mejoras en la calidad del servicio.

Palabras clave: calidad de servicio; SERVQUAL; expectativas; percepciones.

\begin{abstract}
This article starts with the growing relevance that the topic of quality has taken as a strategic imperative to ensure the viability and organizational success. The main purpose is to present the results of the perceived service quality measured in the Guarantee Court of the city of Puerto Montt through the SERVQUAL model. To this end, a questionnaire was collected with 16 indicators, representing the five dimensions indicated by Parasuraman, Zeithaml and Berry, measured on a Likert scale of 5 points, which was applied to people over 18 who were users of the Court of Guarantee on the dates of December 2013 and June 2018. The highest levels of expectations were found in the dimensions: reliability, security, and tangible aspects, while those perceived as having the highest quality were empathy and tangible aspects.In general, service quality levels were unfavorable or negative, mainly because the user's expectations for their perceptions were not exceeded. This information is valuable because it can be used by the organization to improve those attributes of greater relevance to users.
\end{abstract}

Keywords: service quality; SERVQUAL; expectations; perceptions.

Información del manuscrito:

Fecha de recepción: 05 de noviembre de 2020.

Fecha de aceptación: 14 de diciembre de 2020.

Fecha de publicación: 21 de diciembre de 2020. 


\section{Introducción}

Los resultados referidos por (Cabana et al., 2015) reafirman a la calidad cada vez más, como un determinante de la eficiencia y la eficacia en las operaciones comerciales. La mejora de la calidad es el factor principal que afecta la satisfacción del consumidor (A. Meesala and J. Paul, 2016). Al reflexionar en este sentido Kumar (2018) resalta que es más difícil evaluar la calidad de los servicios con respecto a los bienes, saber lo que espera el usuario es el primer paso y, posiblemente, el más crítico. En este sentido, (Vera \& Trujillo, 2018) y (Rezaei, 2018) señalan que para poder mejorar la calidad en un servicio resulta esencial tener claro qué es y cómo medirla.

En este sentido, Parasuraman (1988), proponen un instrumento de medida denominado SERVQUAL, para evaluar la calidad del servicio. Entre los métodos existentes, ha sido aceptado y utilizado en numerosos contextos, demostrando así que puede ser una herramienta eficaz (Jebraeily et al., 2019). Considera un análisis de encuesta basado en la evaluación de la calidad del servicio percibida $y$ esperada por parte de los clientes Demir (2018). Creadas de manera genérica para cualquier tipo de organización, y además sólo recogen la percepción del cliente en un momento específico del tiempo (Martins et al., 2015). (Bustamante et al., 2019) refiere que es, el modelo más aplicado en América Latina, dada su característica flexibilidad interna y adaptabilidad externa de las dimensiones, las cuales pudieron ser perfectamente ajustadas a diversos contextos de servicio y organizacionales.

Cuba cuenta con 29 normas de ISO adoptadas en el campo de los servicios y no se trabaja con ellas debidamente. Por sus características, los servicios revisten ciertas implicaciones para la gestión de la calidad que se deben tomar en cuenta para la ejecución de sus procesos consustanciales. El Ministerio del Turismo se encuentra inmerso en elevar sus índices de calidad, ofreciendo seguridad para los visitantes, ampliando los niveles de oferta, así como diversidad donde lo autenticidad y la cubanía prevalezca.

En el Hotel Meliá Cayo Coco los niveles de calidad en los últimos 
años se han venido afianzando de manera positiva, así lo muestra el aumento del índice de repitencia en los clientes que nos visitan, así como el reconocimiento entre los hoteles de la cayería, a nivel nacional e internacional. Esto se debe en gran medida a los trabajos inversionistas realizados en la planta hotelera, ejemplo de ello, es la reparación capital efectuada en nuestro restaurante Buffet, el bar de la playa y de igual manera el restaurante italiano, no obstante, tanto en materia de técnica como en materia de servicio se hace necesario seguir mejorando.

Si bien se considera que los temas de calidad del servicio reciben un regular tratamiento y análisis en los Consejos de Dirección de la entidad, no se observan soluciones lógicas y científicamente argumentadas que permitan calificar los resultados que desde el punto de vista del cumplimiento de las expectativas alcanzan los clientes relacionado con los servicios de Alimentos y Bebidas ( $A$ y $B$ ), reflejado en las últimas encuestas, que además no se implica en ellas a todos los niveles, ni la totalidad de las áreas. Esto provoca que algunas de las acciones trazadas en materia de calidad no puedan cumplir con su propósito ni ser evaluadas, o medidas, lo que obstaculiza el ajuste en lo posible del servicio ofrecido a las expectativas de los clientes.

El objetivo general de esta investigación es proponer un plan de mejora de la calidad del servicio de Alimentos y Bebidas en el Hotel Meliá Cayo Coco, para ello, proveer a la empresa de una herramienta que le permita tomar decisiones a partir de la percepción y expectativa del cliente sobre el servicio.

\section{Metodología (Materiales y métodos)}

En el estudio se aplicó la escala SERVQUAL con el fin de contribuir a reducir las insuficiencias en la gestión de la calidad de esta entidad, que limitan el cumplimiento de su misión. Se determinó el tamaño final de la muestra, tomando en cuenta el valor mensual más alto correspondiente a la cantidad de clientes que accedieron al servicio, durante el trimestre precedente al mes fiscal objeto de evaluación, que resultó 13669 clientes en el mes de marzo, incluido en el trimestre previo al mes de abril. El tamaño de la 
muestra se obtuvo mediante la ecuación estadística para poblaciones finitas (ecuación 1), asumiendo un nivel de confianza del $95 \%$ y un margen de error del $5 \%$, así como unas variabilidades tanto positivas como negativas del $50 \%$, respectivamente (Aguilar, 2005):

$n=\frac{Z^{2} \mathrm{pqN}}{E^{2}(\mathrm{~N}-1)+\mathrm{pq} Z^{2}}$

En esta ecuación,

$\mathrm{n}=$ Tamaño de la Muestra

$Z$ = valor crítico correspondiente al nivel de confianza elegido, $95 \%=$ 1,96

$\mathrm{p}=$ Variabilidad Negativa $=50 \%$

$q=$ Variabilidad Positiva $=50 \%$

$\mathrm{N}=$ Población $=440$

$E=$ Error $=5 \%=0.05$

Definido el tamaño de la muestra, se aplicaron

374

encuestas

aleatoriamente de doble entrada, quienes a través de una escala de Likert de 1 al 5, (Anderson et al., 2018), indicaron sus expectativas de conformidad para cada elemento de satisfacción con segmentos de respuestas totalmente en desacuerdo, desacuerdo, indiferente, de acuerdo y totalmente de acuerdo 1234 5. De igual, se le solicita al encuestado que indique, el nivel esperado de desempeño, correspondiente a muy alto, alto, medio, bajo; y muy bajo 54321 . Las dimensiones del modelo se elaboraron a partir de los propuestos por (Vera \& Trujillo, 2017) y (Causado et al., 2019), tal como se muestra en la tabla 1.

Tabla 1. Dimensiones e ítems del modelo ServQual aplicados

\begin{tabular}{|c|c|}
\hline Dimension & Ítems de Evaluación por Dimensión \\
\hline \multirow{4}{*}{ Fiabilidad } & $\begin{array}{l}\text { 1.Cuando el Servicio de A y B se compromete a hacer algo en un } \\
\text { tiempo determinado, lo cumple. }\end{array}$ \\
\hline & $\begin{array}{l}\text { 2.Cuando un cliente tiene un problema el personal de A y B muestra } \\
\text { sincero interés por resolverlo. }\end{array}$ \\
\hline & $\begin{array}{l}\text { 3.El personal de A y B desempeña el servicio de manera correcta a } \\
\text { la primera vez. }\end{array}$ \\
\hline & $\begin{array}{l}\text { 4.El departamento de A y B mantiene informados a los clientes sobre } \\
\text { el momento en que se van a desempeñar los servicios. }\end{array}$ \\
\hline \multirow{3}{*}{ Responsabilidad } & $\begin{array}{l}\text { 5.Los empleados de A y B brindan el servicio con prontitud a los } \\
\text { clientes. }\end{array}$ \\
\hline & $\begin{array}{l}\text { 6. Los empleados de A y B siempre se muestran dispuestos a ayudar } \\
\text { a los clientes. }\end{array}$ \\
\hline & $\begin{array}{l}\text { 7.Los empleados de A y B nunca están demasiado ocupados como } \\
\text { para no atender a un cliente. }\end{array}$ \\
\hline \multirow{2}{*}{ Seguridad } & 8.El comportamiento de los empleados de A y B inspira confianza. \\
\hline & 9.Al recibir el servicio de $\mathrm{A}$ y $\mathrm{B}$ el cliente se siente seguro. \\
\hline
\end{tabular}




\begin{tabular}{|c|c|}
\hline & $\begin{array}{l}\text { 10.Los empleados del departamento de A y B tratan a los clientes } \\
\text { siempre con cortesía. }\end{array}$ \\
\hline & $\begin{array}{l}\text { 11.Los empleados de A y B cuentan con el conocimiento para } \\
\text { responder las consultas de los clientes sobre servicio. }\end{array}$ \\
\hline \multirow{3}{*}{ Empatía } & $\begin{array}{l}\text { 12.El Departamento de A y B cuenta con empleados que les brindan } \\
\text { atención personal a los clientes. }\end{array}$ \\
\hline & $\begin{array}{l}\text { 13.El personal de A y B se preocupa de cuidar los intereses de sus } \\
\text { clientes. }\end{array}$ \\
\hline & $\begin{array}{l}\text { 14.Los empleados de A y B entienden las necesidades específicas } \\
\text { de los huéspedes. }\end{array}$ \\
\hline \multirow{5}{*}{$\begin{array}{l}\text { Bienes } \\
\text { Tangibles }\end{array}$} & $\begin{array}{l}\text { 15.La apariencia de los equipos de Servicio Gastronómico es } \\
\text { moderna. }\end{array}$ \\
\hline & $\begin{array}{l}\text { 16. Las instalaciones físicas donde se ofrece servicio de A y B son } \\
\text { visualmente atractivas. }\end{array}$ \\
\hline & 17.La presentación de los empleados de A y B es buena. \\
\hline & $\begin{array}{l}\text { 18.Los materiales asociados con el servicio de A y B (como folletos o } \\
\text { catálogos) son visualmente atractivos. }\end{array}$ \\
\hline & 19.Los horarios de servicios de A y B en el hotel son convenientes. \\
\hline
\end{tabular}

Fuente: Autores (2020).

El valor de las percepciones para cada elemento de satisfacción se obtiene a través del cálculo de la moda. Una vez evaluados los 19 ítems, los autores operativizaron el nivel de calidad de servicio mediante la (ecuación 2) utilizada en (Vicens, 2008), que proporciona tres posibles respuesta: a) que la diferencia entre las percepciones sobre el desempeño $(\mathrm{P})$ y las expectativas (E) $(P>E)$, implica buena calidad; b) que la diferencia sea negativa $(\mathrm{P}<\mathrm{E})$, lo que genera una situación de mala calidad; c) que ambos valores sean iguales $(\mathrm{P}=\mathrm{E})$, y por tanto en esta situación el cliente recibe aquello que espera.

$\mathrm{Q}_{\mathrm{i}}=\sum_{\mathrm{j}=1}^{\mathrm{K}}\left(\mathrm{P}_{\mathrm{ij}}-\mathrm{E}_{\mathrm{ij}}\right)$

Donde:
$\mathrm{Qi}=$ Calidad percibida del objeto $\mathrm{i}$

$\mathrm{K}=$ Número de atributos (19)

P ij = Percepciones sobre el atributo j del objeto i

$E \mathrm{ij}=$ Expectativas sobre el atributo $\mathrm{j}$ del objeto i

El plan de mejoras se elabora en base a los resultados del modelo, a través de la Técnica del Grupo Nominal; para ello, se recomienda seleccionar un grupo de entre cinco y diez integrantes aproximadamente, de manera que se pueda alcanzar consenso (Chang Lau, 2003). En consecuencia, esta etapa es desarrollada por el director, el maître, el sub-maître y seis capitanes, con el objetivo de lograr la implicación necesaria de estas instancias con el plan y la 
responsabilidad de su puesta en marcha. Funge como facilitador el Director de Calidad del Hotel Meliá Cayo Coco.

\section{Resultados (análisis e interpretación de los resultados)}

Aplicada la escala SERVQUAL, con todas las técnicas previstas, se obtuvieron los resultados del estudio. De esta forma, la evaluación de la calidad del servicio global, así como de sus dimensiones en el Hotel Meliá Cayo Coco, se ilustran en la tabla 2.

Tabla 2. Evaluación global y por dimensiones de la calidad del servicio de A y B del Hotel Meliá Cayo Coco

\begin{tabular}{|l|c|c|c|l|}
\hline $\begin{array}{l}\text { Dimensiones de la calidad del } \\
\text { servicio de A y B del Hotel Meliá } \\
\text { Cayo Coco }\end{array}$ & $E>P$ & $E=P$ & $E<P$ & $\begin{array}{l}\text { Evaluación } \\
\text { de la } \\
\text { calidad }\end{array}$ \\
\hline 1. Fiabilidad & 2 & 2 & 0 & mala \\
\hline 2. Responsabilidad & 2 & 1 & 0 & mala \\
\hline 3. Seguridad & 1 & 2 & 1 & buena \\
\hline 4. Empatía & 0 & 3 & 0 & buena \\
\hline 5. Bienes Tangibles & 3 & 2 & 0 & mala \\
\hline $\begin{array}{l}\text { Calidad Global del Hotel Meliá Cayo } \\
\text { Coco }\end{array}$ & 8 & 10 & 1 & mala \\
\hline
\end{tabular}

Fuente: Elaboración propia (2020).

A pesar de alcanzar las encuestas alto grado de conformidad, mostrado por los indicadores de totalmente de acuerdo (11 ítems) y el resto (8 ítems) de acuerdo, la mayoría de las dimensiones con $\mathrm{P}<\mathrm{E}$ es inferior a lo esperado y ello implica insatisfacción del cliente. Se reconoce a la vez, que por las características del servicio que se presta en un hotel 5 estrellas es difícil superar las expectativas de los clientes, pero no debe suceder que perciban un servicio inferior al esperado.
La ejecución de esta técnica permitió que de manera organizada se expusieran, discutieran y seleccionaran jerárquicamente, acciones para alcanzar mejoras en la calidad del servicio del Hotel Meliá Cayo Coco, a partir plan de mejoras las que se presentan a continuación por cada una de las dimensiones.

\section{- Acciones correctivas}

\section{Fiabilidad}

- Revisar el bloque habitacional con el fin de eliminar vestigios de 
cartas de vinos, desactualizadas

y de servicios que en estos momentos no se brindan.

\section{Responsabilidad}

- Lograr que cuando un trabajador recibe una queja este trate de darle todo el seguimiento posible, hacer propia la queja, para que el cliente pierda la menor cantidad de tiempo posible.

- Hacer una correcta distribución del trabajo y los trabajadores por cada uno de los puntos de venta permitiendo que se trabaje en todos los sitios de forma balanceada.

\section{Seguridad}

- Incluir los resultados de la evaluación de auto, preparación de los empleados en cortesía y normas de servicio a la evaluación mensual del desempeño, vinculada a los pagos por estimulación.

\section{Bienes tangibles}

- Reparar los equipos de refrigeración rotos en el hotel.

- Incluir en el plan de inversiones la reparación del ranchón de la playa y la climatización del restaurante Caletas, en aras de eliminar quejas por presencia de mosquitos.
- Incrementar y actualizar los materiales asociados con el servicio en aras de que estos sean más atractivos visualmente.

\section{- Acciones preventivas}

\section{Fiabilidad}

- Crear un stock de productos claves en los puntos de venta de A y $B$ que permita paliar situaciones especiales que se presentan en el servicio.

- Incrementar los niveles de comunicación entre los diferentes departamentos con el fin de no brindar información a los clientes que no se pueda cumplir en el tiempo requerido.

- Disminuir en lo posible las ocasiones en que están cerrados los restaurantes especializados y aumentar las capacidades de estos en temporada baja.

\section{Responsabilidad}

- Desarrollar acciones de capacitación que permitan elevar y valorar la importancia que tienen los clientes para nuestro hotel y para el país en general.

- Seguir fomentando el valor trabajo en equipo el que permite 
que trabajadores de un punto de venta apoyen en diferentes horarios a otros que lo necesitan.

\section{Seguridad}

Desarrollar acciones de capacitación que muestren temas de cortesía y su importancia para el hotel y el país en general.

\section{Conclusiones}

1. El estudio teórico acerca de la gestión de la calidad es el proceso que identifica y proporciona información útil y descriptiva acerca del valor del desempeño de la organización en la prestación de servicios, con el fin de contribuir en el proceso de toma de decisiones.

2. La confección de un plan de mejora de la calidad en la prestación de los servicios de alimentos y bebidas demanda el diseño de acciones que responda a la proyección de resultados sobre la base de potenciar la organización y control de los procesos operativos que garantice la satisfacción del cliente.

3. El desarrollo de la evaluación de la calidad con características de enfoque al cliente, sistematicidad, implicación y compromiso de los empleados y directivos contribuye a reducir las insuficiencias de la gestión de la calidad y con ello la obtención de resultados coherentes con la misión del Hotel Meliá Cayo coco.

\section{Bibliografía}

Aguilar, S. (2005). Fórmulas para el cálculo de la muestra en investigaciones de salud. Salud en Tabasco, 11, 338, Article 1-2.

Anderson, D. R., Sweeney, D. J., Williams, T. A., Camm, J. F., \& Cochran, J. J. (2018). Statistics for business \& economics. Cengage Learning.

Bustamante, M., Zerda, E., \& Francisco, M. (2019). Fundamentos de calidad de servicio, el modelo Servqual. Revista Empresarial, 13, 15, Article 2. https://doi.org/DOI: 10.23878/empr.v13i2.159

Cabana, V., Segundo, R., Gálvez, P., \& Muñoz, C. (2015). Variables críticas en las ventajas competitivas de restaurantes gourmet, La Serena, Chile. Cuadernos de Administración, 31, 67, Article 54.

Causado, E., Charris, A., \& Guerrero, E. (2019). Mejora Continua del Servicio al Cliente Mediante ServQual y Red de Petri en un Restaurante de Santa Marta, Colombia. 
Información Tecnológica, 30, 84, Article 2. https://doi.org/http://dx.doi.org /10.4067/S0718-

07642019000200073

Chang Lau, I. C. (2003). Metodología, para la satisfacción del cliente e identificación de sus correspondientes acciones de mejoramiento.

Jebraeily, M., Rahimi, B., Fazlollahi, Z., \& Lotfnezhad, H. (2019). Using SERVQUAL Model to Assess Hospital Information System Service Quality. Hormozgan Medical Journal, 23, 6, Article 1. https://doi.org/ 10.5812/hmj.86977.

Martins, A., Crespo , J., Ramos, T., \& Fael, J. (2015). Assessing Obstetrics Perceived Service Quality at a Public Hospital. Procedia - Social and Behavioral Sciences 181, 422.

https://doi.org/10.1016/j.sbspr 0.2015 .04 .904

Meesala and J. Paul. "Service quality, consumer satisfaction and loyalty in hospitals: Thinking for the future". Journal of Retailing and Consumer Services. Vol. 40, pp. 261-269. 2018. https://doi.org/10.1016/j.jretco nser.2016.10.011

M. Kumar, K. Sujit and V. Charles. "Deriving managerial implications through
SERVQUAL gap elasticity in UAE banking", International Journal of Quality \& Reliability Management, Vol. 35 Issue 4, pp. 940-964. 2018. DOI: 10.1108/IJQRM-10-2016-

0176. ISSN: 0265-671X https://doi.org/10.1108/IJQR M-10-2016-0176

Parasuraman, V. Zeithaml, and L. Berry. "SERVQUAL: A multiple-item scale for measuring consumer perceptions of service quality". Journal of Retailing. Vol. 64, Issue 1, pp. 12-40. 1988.

P. Demir, M. Gul and A. Fuat Guneri. "Evaluating occupational health and safety service quality by SERVQUAL: a field survey study". Total Quality Management \& Business Excellence. 2018. DOI: 10.1080/14783363.2018.143 3029.

Rezaei, J., Kothadiya, O., Tavasszy, L., \& Kroesen, M. (2018). Quality assessment of airline baggage handling systems using SERVQUAL and BWM. Tourism Management, 66, 93. https://doi.org/10.1016/j.tourm an.2017.11.009

Trujillo, J. V. y. A. (2018). El efecto de la calidad del servicio en la satisfacción del derechohabiente en instituciones públicas de salud en México. Contaduría y Adminis tración63, 22. 
doi:http://dx.doi.org/10.1016/j.

cya.2016.07.003

Vera, J., \& Trujillo, A. (2017). Escala mexicana de calidad en el servicio en restaurantes (EMCASER). INNOVAR.

Revista de Ciencias

Administrativas y Sociales, 27, 59, Article 63.

Vera, J., \& Trujillo, A. (2018). El efecto de la calidad del servicio en la satisfacción del derechohabiente en instituciones públicas de salud en México. Contaduría y Administración 63, 22, Article 2. https://doi.org/http://dx.doi.org /10.1016/j.cya.2016.07.003 